\title{
LAS ALIANZAS PÚBLICO-PRIVADAS COMO GATILLADORAS DE INNOVACIÓN EN LAS ORGANIZACIONES PÚBLICAS: REFLEXIONES A PARTIR DE LA SITUACIÓN CHILENA
}

\author{
Cristián Pliscoff V. Juan Pablo Araya O. \\ cpliscof@iap.uchile.cl_jpabloaraya@iap.uchile.cl \\ Instituto de Asuntos Públicos \\ Universidad de Chile
}

El artículo aborda la problemática de las alianzas público-privadas como arreglos institucionales que pueden generar innovación en la gestión pública. Para esto se examina la experiencia del Programa Inglés Abre Puertas y del Sistema de Concesiones. Estos dos casos permiten, al menos de manera exploratoria, dar cuenta de los principales desafíos en su implementación y de los principales tipos de innovación que las alianzas han logrado.

Palabras Clave: Alianzas público-privadas, innovación, Nueva Gestión Pública. 


\section{PUBLIC-PRIVATE PARTNERSHIPS AS GENERATORS OF INNOVATION IN PUBLIC SERVICE ORGANIZATIONS: THOUGHTS BASED ON THE CHILEAN EXPERIENCE}

This article studies the issue of public-private partnerships as institutional arrangements which may generate innovation within public management. For this purpose two programs are examined: Ingles Abre Puertas (English Opens Doors), and the concession system. These two cases provide information, at least at an exploratory level, on the main implementation challenges and the principal types of innovation they have achieved. Keywords: Public-private partnerships, innovation, New Public Management. 


\section{INTRODUCCIÓN}

Las alianzas público-privadas (APP) se han instalado en el ámbito público como una de las formas en la cual el sector público y el privado se unen para resolver problemas públicos. Este tipo de instrumentos de política tiene diversos orígenes y responde a diferentes marcos teóricos que de una u otra manera lo justifican y/o lo explican (Wettenhal, 2003; Heinrich, Lynn, \& Milward, 2009; Kooiman, 1993). Desde resolver problemas tan específicos como la infraestructura, hasta enfrentar la pobreza de una región o país, o simplemente para aunar fuerzas para resolver problemas de interés colectivo, las alianzas público-privadas han saltado a la palestra pública como un fenómeno característico de las sociedades globales.

El presente artículo busca reflexionar sobre una dimensión particular de las alianzas público-privadas, particularmente la que se refiere a la capacidad que éstas tienen de generar cambios, y, muy especialmente, innovación en la gestión pública. En otras palabras, asumiendo que las APP son una realidad en el contexto actual, se pretende ver en qué medida estos nuevos arreglos institucionales pueden transformarse en un ambiente propicio para estimular, crear y/o generar innovación en la gestión pública. En este sentido, el presente trabajo sigue la línea planteada por Sorensen y Torfing (2011) respecto del desarrollo que han tenido las investigaciones sobre el nacimiento y surgimiento de las redes y las alianzas públicoprivadas, sugiriendo que poco se ha hecho para establecer el nexo entre alianzas e innovación. Para dar cuenta de este objetivo, el presente trabajo se iniciará con una reflexión sobre el tema de las alianzas público-privadas, para luego desarrollar el tema de la innovación en la gestión pública, finalizando con las reflexiones referidas a la relación entre ambas temáticas. Algunas ideas respecto de futuras investigaciones y algunas conclusiones generales son presentadas en la parte final de este documento. 


\section{LAS APP: UN FENÓMENO PROPIO DE UN ESTADO EN CONSTANTE CAMBIO}

Una de las primeras líneas teóricas que se han desarrollado para justificar este tipo de acción pública proviene del debate generado a fines de los ochenta y comienzos de los noventa en torno a la escuela de la Nueva Gestión Pública (Hood, 1991). Para esta escuela, las alianzas público-privadas son concebidas como técnicas de gestión que permiten utilizar de mejor forma los escasos recursos que posee el Estado. En este mismo contexto temporal, Savas (1990) utiliza el análisis de las privatizaciones para incluir las alianzas público-privadas como una forma de injerencia del mercado en el sector público. En este debate, se asume que existen ciertas áreas en que el Estado no posee los recursos ni las capacidades para intervenir, y, por lo tanto, es necesario un acuerdo con el sector privado. Esta visión es muy similar a la planteada por Williamson (1988), quien plantea, a partir de su Teoría de los Costos de Transacción, que dependiendo del tipo de actividad que se quiere abordar, será necesario un diferente acuerdo para disminuir los costos de transacción. Este tipo de justificación de las APP se refiere más bien a un argumento principalmente eficientista, más que una colaboración propiamente tal (Donahue y Zeckhauser, 2011). La Tabla $N^{\circ} 1$ entrega una visión de los distintos tipos de APP que la literatura reconoce. 


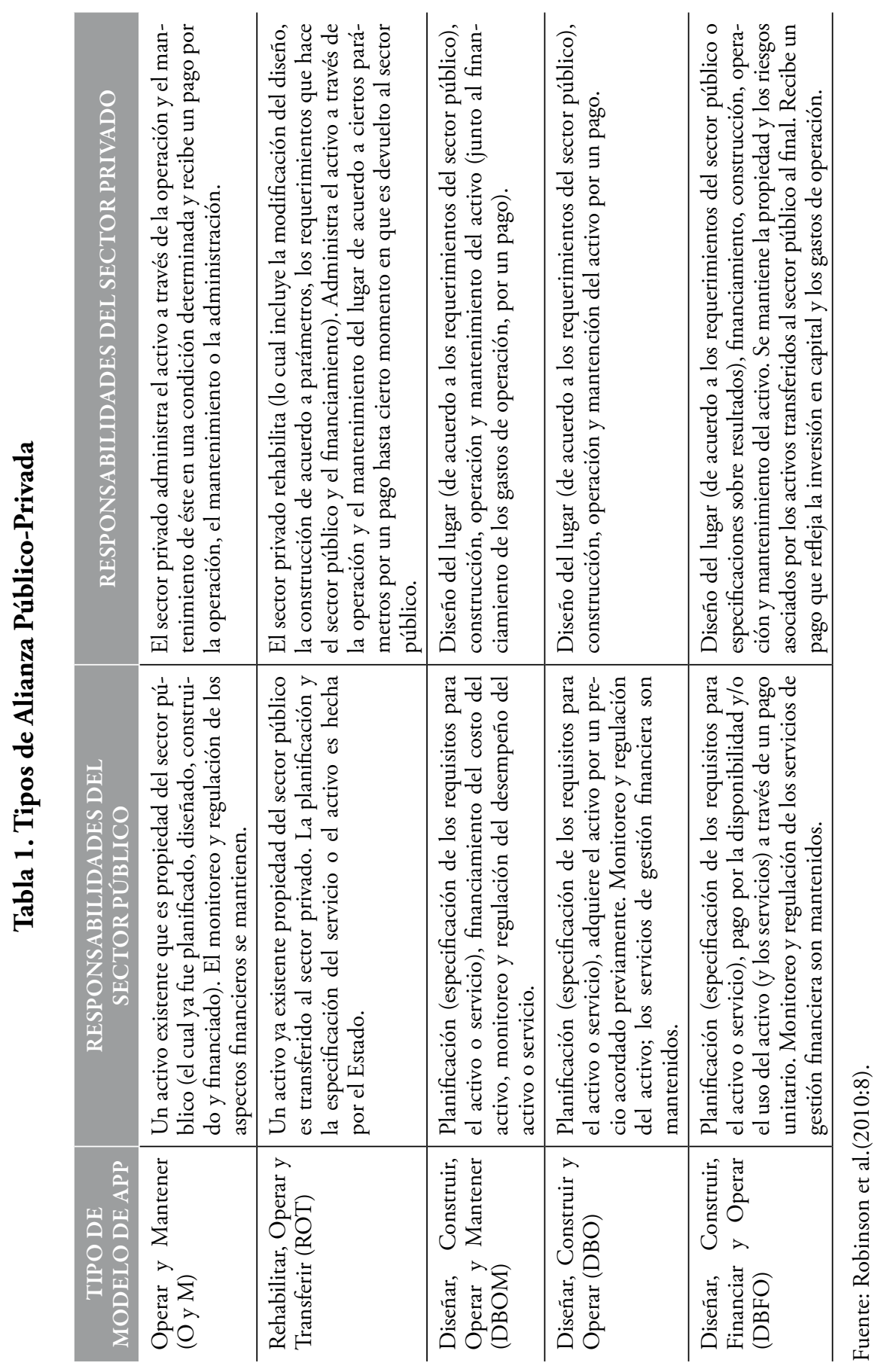


Otra forma de entender este tipo de nuevas expresiones de política surge a partir de la llamada Teoría de la Gobernanza. Este marco teórico, desarrollado extensivamente entre otros por Kooiman (2003), se basa en el análisis de las nuevas articulaciones sociales y al nuevo rol que cumple el Estado en las sociedades globalizadas, por lo tanto es necesario pasar de una comprensión de la actividad pública centrada en la acción monopolica del Estado, a una que apunte a la "dirección" de la sociedad. El Estado, más que proveer servicios o soluciones, actúa junto a otros actores para la entrega de éstos. La noción de gobernanza asume que existen actores de diferentes sectores de la sociedad que deben articularse en pos de la resolución de problemas de carácter público. Del mismo modo se asume que existen "problemas extrańos" (wicked problems) (Koppenjan y Klijn, 2004; Rittel y Webber, 1973) que por su naturaleza ameritan la interacción y el trabajo colaborativo de diferentes tipo de actores. Este concepto es muy similar al que surge de las proposiciones de Castells en su Teoría de la Globalización, toda vez que se asume que el Estado, debido a las nuevas realidades producidas por la globalización, es incapaz de responder de manera adecuada a los nuevos desafíos a los que se ve enfrentado (Castells, 1992).

Desde una perspectiva latinoamericana, la necesidad de pensar en la creación y desarrollo de APP centrada en la realidad guarda relación con la falta de recursos financieros en la administración del Estado, como resultado de las políticas neoliberales que tenían como eje rector el reducir el Estado a su mínima expresión, restringiendo diferentes políticas públicas en materia social (Bresser, 1999). Lo anterior ha sido uno de los principales factores que han propiciado el establecimiento de las alianzas público-privadas en la búsqueda de mejorar el desempeño y el impacto de las políticas públicas en la sociedad.

Desde finales de los noventa, el debate se focalizó en torno a las implicancias de los arreglos organizacionales entre el Estado y el sector privado e incluso el tercer sector, para determinar las consecuencias de casi una década de colaboración a través de instrumentos de acción pública. Un trabajo paradigmático en esta línea de investigación en el caso de la administración pública estadounidense es el trabajo de Milward y Provan (2000). Los autores desarrollan el concepto del "Estado Hueco”, que sería el tipo de Estado que se estaría conformando a propósito del uso intensivo de privatizaciones o "concesiones" a privados u organismos no gubernamentales, para cumplir funciones que históricamente han sido propias del Estado. 
La literatura ha avanzado notoriamente, y junto con ello se ha comenzado a desarrollar investigaciones orientadas a detectar, incipientemente, ciertas regularidades, en el establecimiento de las APP, a través de casos de países desarrollados y en vías de desarrollo, y en diversos sectores de política. En lo que se refiere al sector productivo, Devlin y Moguillansky (2009), a partir de un proyecto de investigación desarrollado en CEPAL en diez países, entregan recomendaciones que pudiesen servir como parámetros para testear el desempeño de las APP en diversos ámbitos de política pública. Los autores plantean once principios a tomar en consideración al momento de diseñar una intervención de este tipo. Por otro lado, el extenso trabajo de Hodge y Greve (eds.) (2005) presenta una colección de investigaciones de diferentes autores, de países desarrollados particularmente, y de distintos sectores de política, que también aportan conclusiones respecto de las características que deberían cumplir los acuerdos público-privados para tener éxito.

Por último cabe señalar que la conceptualización de las APP como la colaboración voluntaria del mundo privado hacia el mundo público (definiendo redes potenciales para abordar problemas públicos conjuntamente), compartiendo riesgos, costes y beneficios para efectos de contribución al bienestar social, se ha organizado en torno al tema de la llamada Responsabilidad Social Empresarial (RSE) (Korhonen, 2003). Según este enfoque, se justifica el porqué se deben sumar los privados a trabajar en la solución de problemas públicos. Como el foco del presente documento es la innovación generada desde el sector público no se desarrollará mayormente este ámbito de discusión teórica, pero sin duda aporta a la discusión más amplia de la temática expuesta en este documento.

\section{LAS APP COMO GATILLADORAS DE INNOVACIÓN EN LA GESTIÓN PÚBLICA}

En cierta medida, la literatura recién señalada plantea que las APP son una innovación en el sector público para poder dar cuenta de nuevas dinámicas sociales existentes. En ese sentido, podríamos verlas como una innovación en sí mismas, toda vez que implican el uso de nuevos dispositivos formales y/o informales para poder actuar en torno a la resolución de una problemática determinada. Pero por otro lado, las APP, en la medida que propician esta nueva forma de hacer las 
cosas, pueden ser vistas como gatilladoras de innovación tanto en el ámbito público como el privado. En el presente documento focalizaremos nuestros esfuerzos hacia la comprensión de las APP como gatilladoras o generadoras de innovación en la gestión pública. Para reconocer estos nexos en mayor detalles se analizarán algunos aspectos sobre el tema de la innovación en general, para luego focalizar en la innovación de la gestión pública.

\section{INNOVACIÓN EN LA LITERATURA DE LAS CIENCIAS DE LA ADMINISTRACIÓN}

Los estudios sobre innovación en las Ciencias de la Administración tienen dos fuentes de origen. La primera, más antigua, proviene desde la macroeconomía a comienzos del siglo xx, que entendía a la innovación como propia de los esfuerzos de los actores económicos por desarrollar nuevos mercados. Posteriormente, a mediados de este mismo siglo, la innovación pasa a ser una actividad mucho más compleja, toda vez que se le entiende como un espacio de ánalisis al que aportan diferentes disciplinas, tales como la economía, la sociología, la política y psicología.

Según Osborne y Brown(2005), existen cuatro elementos en todas las definiciones sobre innovación. En primer lugar, la innovación se relaciona y se refiere a la idea de novedad, vale decir, con el primer uso de un nuevo conocimiento dentro de una organización. Un segundo elemento es la idea de invención, relacionada con la generación de una nueva idea y su implantación dentro del proceso organizacional o un producto. Un tercer concepto, es la idea de proceso y resultado, particularmente, en lo que respecta a la idea de discontinuidad en éstos. Siguiendo con esta misma línea, un cuarto elemento que los autores señalan es la idea de cambio y continuidad. Tanto el desarrollo organizacional como la innovación generan como resultado cambios significativos en la configuración de la organización. Sin embargo, la diferencia sustancial entre cambio organizacional e innovación es que el primero ocurre dentro de un paradigma determinado, mientras que la innovación implicaría un quiebre de paradigma, generando algo nuevo. Como producto de este nuevo paradigma se altera la naturaleza del producto/servicio y/o mercado, 
generando una discontinuidad. En función de lo anterior, estos autores definen innovación como “(...) la introducción de novedad en un sistema usualmente, no siempre, en términos relativos, y por la aplicación (y ocasionalmente invención) de una nueva idea. Esto produce un proceso de transformación que genera una discontinuidad en términos del sujeto mismo (como un producto o servicio) y/o su medio ambiente (como una organización, mercado o comunidad)" (Osborne y Brown, 2005:116).

En esta misma línea de análisis y tal como lo señala Constidine et al. (2009), la discusión sobre innovación se puede agrupar en torno a tres tipos de preguntas: la pregunta respecto del proceso o el producto, la pregunta sobre cambios incrementales o radicales, y finalmente la pregunta sobre lo normativo de la innovación. Respecto de la pregunta sobre el proceso o el producto, se puede señalar que la innovación puede referirse tanto a los productos que se crean o respecto de los procesos previos a los productos. Este aspecto es particularmente relevante en el sector público, toda vez que muchas innovaciones se dan en torno a la asignación o reasignación de recursos, lo que se traduce muchas veces en diferentes e innovativos procesos. La segunda pregunta respecto del tipo de cambio al que se refiere la innovación guarda relación con el tipo de cambio que podemos señalar como "innovador". Siguiendo con la definición dada en el párrafo precedente, la innovación se da más bien en torno a cambios radicales, toda vez que implican novedad y discontinuidad con lo anterior. Finalmente, el elemento normativo de la innovación guarda relación con la supuesta predisposición a aceptar todo tipo de innovación como positiva, lo cual supone a veces la idea de que la innovación es buena en sí misma. No cabe duda que la literatura de las Ciencias de la Administración ha superado en gran medida esta fe ciega en la innovación, y se ha preocupado de darle un sentido y un contexto para hacerla pertinente y útil.

Osborne y Brown (2005), exponen una tipología de innovación de acuerdo a la naturaleza de esta. En la Tabla No 2 se resumen estas categorías. 


\section{Tabla No 2. Clasificación de la naturaleza de la Innovación}

\begin{tabular}{l|c}
\multicolumn{1}{c|}{\begin{tabular}{c}
\multicolumn{1}{c|}{ NATURALEZA DE } \\
LASIFICACION
\end{tabular}} & AUTORES CLAVES \\
$\begin{array}{l}\text { Como impulso de investigación o impulso del } \\
\text { mercado }\end{array}$ & Burns and Stalkers (1961) \\
\hline $\begin{array}{l}\text { Como producto de la crisis o bajo rendimiento } \\
\text { organizacional }\end{array}$ & Cyert and March (1963) \\
\hline $\begin{array}{l}\text { “Cui bono" o a quién beneficia y cómo } \\
\text { Como innovación en un producto o proceso }\end{array}$ & Daft and Becker (1978) \\
(y sus variantes) & Aon Hippel (1978, 1982) \\
\hline $\begin{array}{l}\text { Por la relación entre el impacto de una innova- } \\
\text { ción sobre su contexto organizacional y sobre el } \\
\text { mercado en un sentido amplio }\end{array}$ & $\begin{array}{c}\text { Zalman et al. (1973) } \\
\text { Bessant and Grunt (1985) }\end{array}$ \\
\hline
\end{tabular}

Fuente: Osborne \& Brown (2005:126)

\section{INNOVACIÓN EN LA LITERATURA DE LA GESTIÓN PÚBLICA}

En el imaginario colectivo ha existido la idea de que las grandes organizaciones públicas son resistentes a la innovación. Según Borins, esto se produciría por cuatro factores que obstaculizan la innovación en los servicios públicos: el contexto político, que orienta el fin de la actividad pública en intereses políticos centrados en el poder, más que en el mismo sistema organizacional; la necesidad de los servicios públicos de restringir la flexibilidad individual en beneficio del accountability; el escrutinio de los medios de comunicación en los errores de los servidores públicos, y; la falta de incentivos para la innovación en los servicios públicos (Borins, 2001).

Todos estos factores nos indicarían que la innovación es un fenómeno; por decir lo menos, escaso y muy difícil de alcanzar, pero a pesar de ello, la innovación existe y es reconocida como tal. Nuevos servicios se producen, nuevas ideas se implementan y nuevos actores se suman a la prestación de servicios. 
Entonces, ¿̨cuáles son los factores que explicarían la aparición de innovación en la gestión pública? A este respecto podemos reconocer dos grandes trabajos que aglutinan los hallazgos en la literatura académica. Primero podemos señalar el trabajo de Newman et al. (2001:62), quienes indican que la innovación en la gestión pública es el producto de una compleja interacción de condiciones conductuales, culturales, estructurales y del medio ambiente. A ello suman las cualidades y los valores individuales de actores clave; demandas de cambio generadas desde el medio externo (producidas tanto por ciudadanos, usuarios de los servicios o gobiernos por igual); procedimientos institucionales y cultura organizativa; y por redes tanto internas como externas, todos jugando un rol crítico en la configuración y naturaleza, y el ámbito de la innovación” (Considine, et al., 2009:27). En esta definición se pone de manifiesto que la innovación en la gestión pública es un proceso complejo, donde muchos factores y situaciones tienen que darse para permitir o facilitar la innovación.

El segundo aporte que entrega elementos para reconocer el cómo surge la innovación, es el trabajo de Osborne y Brown (2005), quienes aglutinan la literatura en esta materia en cuatro grandes vertientes. Un primer enfoque relaciona la innovación con el ciclo de vida de los servicios públicos. Particularmente, la innovación se relacionaría con cambios de gobierno o de liderazgo lo que acarrea la adopción de nuevas ideas, que pudiesen gatillar innovaciones. Un segundo enfoque relaciona la innovación dentro del rol de la gestión estratégica y la planificación. En este sentido, y tomando nuestra definición de innovación, cuando la planificación estratégica genera como resultado la necesidad de generar novedad, o en otro términos un cambio no incremental, en los servicios o procesos, se estaría generando innovación. La tercera visión señala que la innovación surge como necesidad de contrarrestar la naturaleza burocrática de los servicios públicos. Esto porque la misma naturaleza burocrática de la organización pública se transforma en una piedra de tope para la innovación, entonces, si algunas de estas prácticas se modifican, se pudiese dar lugar a innovación. Por último, la innovación se entendería como una búsqueda por la eficiencia (Baldock y Evers, 1991). 


\section{¿QUÉ GATILLA LA INNOVACIÓN EN UN SERVICIO PÚBLICO?}

Dentro del análisis expuesto en la literatura se pueden destacar los siguientes aspectos. Primero, el emprendedor o gestor de la innovación juega un rol clave para estimular la innovación. El "héroe de la innovación" tiende a ser un individuo "optimista, con una fuerte orientación a resultados y dispuesto a tomar riesgos" (Considine et al, 2009). Esta línea de análisis es muy similar al desarrollo teórico que hace Moore cuando le asigna al directivo público un rol esencial en la innovación para crear valor público (Moore, 1995). En el caso chileno por ejemplo se habló, particularmente en la década de los 90, de un grupo de "intra emprendedores" que lideraron procesos no solo de cambio organizacional, sino de innovación, específicamente en el Servicio de Impuestos Internos, el Registro Civil o el Fondo Nacional de Salud (Fonasa) (Waissbluth, 2005).

El segundo elemento del análisis se refiere a las presiones externas por innovación. De acuerdo a Walker (2006) el ambiente del sector público está marcado por un constante cambio y dinamismo que genera la necesidad por el cambio y la innovación. Un ambiente marcado por los ciclos políticos, por los cambios en las tecnologías, las presiones de los ciudadanos, organizados o no, y por los constantes cambios económicos, normativos y administrativos, hace que el ambiente externo sea una fuente constante de presiones para la innovación. Sin embargo, tal como fue planteado respecto de la realidad de los gobiernos locales, las presiones de la ciudadanía, pueden devenir en un obstáculo para la innovación, cuestión que nos lleva a plantear que eventualmente los otros factores externos, también pueden ser vistos como obstáculos para futuras innovaciones. En este sentido, desde el punto de vista de esta investigación, lo relevante es que se debe incorporar el análisis de los factores que determinan la innovación a los factores externos, y una vez reconocido este hecho, determinar qué factores estimulan o evitan la innovación. En este sentido, podemos incorporar otra fuente teórica que nos entrega antecedentes para poder entender cómo se gatilla la innovación en el sector público a partir de la teoría de redes, entendiendo que una red determina el contexto en el que se desarrolla una organización. En particular, esta teoría viene a quebrar con las teorías elitistas o estructuralistas de toma de decisiones, centradas en grupos o estructuras cerradas, y nos ayuda a comprender que el juego político, y el propio diseño de políticas públicas se da respecto de las redes que se articulan. En esta literatura se plantea que los "lazos fuertes" pueden no ser tan significativos desde 
el punto de vista de las redes como sí lo serían los "lazos débiles" (weak ties) (Granovetter, 1973). En este sentido, una organización que forma parte de una red, a la cual se le suman estos últimos tipos de lazos, podría verse sujeta a un tipo nuevo de influencia, que pudiese estimular la necesidad de innovar.

El tercer asunto se refiere a los factores internos que facilitan la innovación. El trabajo de Damanpour (1991, citado en Constidine et al., 2009:34), señala que la innovación "está positivamente relacionada con un alto nivel de especialización de la organización, con altos niveles de diferenciación funcional, altos niveles de profesionalismo, una actitud positiva al cambio de parte de los niveles gerenciales, altos niveles de recursos de conocimiento técnico, intensidad administrativa (vale decir, la presencia de una alta proporción de gerentes), con la disponibilidad de recursos libres, y en organizaciones con redes de comunicaciones internas y externas muy intensas". Estos factores nos ayudan entonces a determinar qué tipo de organizaciones son más proclives a la innovación y cuáles no, de acuerdo a las características que éstas posean.

Finalmente, los marcos teóricos ya señalados previamente, están referidos necesariamente a los marcos normativos de cada uno de los individuos que forman parte de las organizaciones estudiadas. En este sentido, de acuerdo a los tipos de "conceptos o nociones" que se manejen sobre la innovación, podremos facilitarla. De esta forma, se puede asumir que la innovación es un concepto socialmente construido y que el éxito o el fracaso en su diseño o implementación, puede verse afectado, no solo por los tres factores analizados previamente, sino también por lo que cada sociedad, cultura u organización entienda por innovación. Entonces, una innovación se podría llegar a gestar si existen individuos con marcos normativos similares con una propensión al cambio.

Por otro lado, Osborne y Brown (2005), reconocen tres modelos que explicarían cómo se inicia la innovación en los organismos públicos. El modelo del Innovador Individual pone el foco en la persona como agente de innovación en un servicio público. El innovador tiene en esencia una mentalidad de empresario o emprendedor, quien es capaz de promover innovación por determinadas cualidades personales que posee. Robert y King (1996) señalan que alguna de estas características que posee son: tenacidad, trabaja muchas horas, es orientado a resultados, con voluntad para tomar riesgos, confidente y hábil en utilizar las conexiones 
políticas. Aunque este es uno de los enfoques más señalados en la literatura, adolece de no considerar el contexto de las organizaciones públicas, ni considerar la sustentabilidad a largo plazo de la innovación.

Un segundo enfoque se centra en un modelo estructural. Este reconoce la influencia de los individuos, pero acentúa el contexto organizacional por sobre la personalidad como factor gatillante de la innovación. En este sentido, según Barlett y Dibben (2002), existen roles dentro de la organización que se necesitan para que la innovación sea efectiva. En primer lugar el rol de Innovation Champion se divide dentro de dos sub-roles: el public champion, quien patrocina la innovación con una motivación de mejorar la calidad de los servicios públicos y el empowered champion, quien patrocina la innovación por una motivación centrada en la realización personal. En segundo lugar, el rol del Innovation Sponsor se relaciona con alguien que en el contexto político del servicio público provee el mandato político para poder llevar a cabo la iniciativa de innovación.

En tercer lugar, el Enfoque Contingente analiza la interacción del innovador al interior del servicio público y el ambiente organizacional más amplio. Borins (2001) señala que no siempre los innovadores son los directivos en los servicios públicos, sino que también provendrían de diferentes áreas del espectro organizacional. En este sentido, la innovación se produciría porque existen incentivos como políticas claras desde el nivel gubernamental, nuevos liderazgos, crisis organizacional, problemas organizativos internos y/o nuevas oportunidades para crecer. Por ejemplo, caso interesante en esta línea fue el trabajo desarrollado en la Subsecretaría de Desarrollo Regional y Administrativo del Ministerio del Interior chileno, que desarrolló un programa de estímulo a la innovación dirigido a funcionarios públicos municipales. Este grupo de innovadores provino principalmente de los cuadros administrativos y profesionales (Torres, 2004). 


\section{Alianzas público-PRIVAdas e inNOVAción EN LA GeStión pública CHILENA}

\section{El Sistema de CONCESIONES CHILENO COMO APP GATILLANTE DE INNOVACIÓN EN PROVISIÓN DE INFRAESTRUCTURA PÚBLICA}

El caso chileno de concesiones en infraestructura es un ejemplo de las alianzas público-privadas como detonantes de innovación en la gestión pública. En breve, el modelo de concesiones chileno adopta la forma conocida como Building, Operate and Transfering-BOT- (ver Tabla 1 de este artículo), donde básicamente una empresa privada construye, financia y opera un proyecto de infraestructura, cobra por el uso de los servicios asociados a este por un período prefijado luego de este período, transfiere la infraestructura al sector público. Solamente por concepto de rutas, autopistas urbanas y aeropuertos, entre 1992 y 2006 la inversión en concesiones ascendió a USD\$ 6.917 millones, ubicando a Chile entre los primeros puestos en materia de inversión privada en el sector público en infraestructura (Bitran y Villena, 2010).

A comienzos de la década de los '90, la infraestructura pública en Chile presentaba una situación de déficit y la que existía se encontraba en estado de deterioro, por lo cual se necesitaba gran cantidad de recursos financieros para subsanar esta problemática y asegurar el crecimiento económico del país. Antes de los '90, las concesiones se normaban por el DFL No 591 del año 1982. Bajo esta normativa no se adjudicó ningún proyecto de concesiones, ya que la problemática de infraestructura pública no era un problema instalado en la agenda de decisiones. En particular, la participación del sector privado en el público se reflejaba básicamente en la privatización de empresas públicas. (Bitran y Villena, 2010; Blazevic, 2008).

Con la vuelta a la democracia, se toma conciencia sobre la ineficacia del antiguo sistema tradicional de ejecución de obras públicas en materia de infraestructura. Por esta razón, el presidente Aylwin instruye al ministro de Obras Públicas de la época, Carlos Hurtado, el análisis para el establecimiento de una estrategia de infraestructura pública mediante la asociatividad público-privada. A diferencia de los ańos '80, existe un consenso en los actores políticos de la insuficiencia en infraestructura y el impacto de esto en la proyección de crecimiento económico 
del país. Según Carlos Hurtado, "Se contó con apoyo de gente que había pensado estos temas en la Cámara Chilena de la Construcción, con el respaldo desde luego del Presidente de la República, Patricio Aylwin. Un mérito indiscutible también fue el del Congreso. Tuvimos la suerte de contar con el apoyo de muchos parlamentarios de los más variados sectores, los que dieron su respaldo debido al mérito de haber tenido "el tino y la buena leche" de atraer positivamente a la gente hacia esto y no entrar en peleas inútiles y estériles" (citado en Blazevic, 2008:21).

Básicamente existió acuerdo político en los siguientes puntos:

- La insuficiencia de recursos públicos para enfrentar la escasez en infraestructura pública;

- El establecimiento de alianzas público-privadas en el financiamiento y gestión de la infraestructura permitiría reducir este déficit;

- La experiencia internacional respaldaba la eficacia de estos arreglos institucionales para resolver los déficits en infraestructura;

- El establecimiento de alianzas público-privadas permitiría liberar recursos del Estado para otras inversiones de alto impacto social. Según Ricardo Lagos, ex ministro de Obras Públicas, lo que no se gastó en concesiones se pudo gastar en infraestructura social, aquellas que si no las hace el Estado no las hace nadie (Blazevic, 2008:21).

El inicio de una política mediante la cual se incorporaba capital privado en infraestructura pública, fue el inicio de una gran variedad de iniciativas entre el Estado y el sector privado para la provisión de bienes públicos en diferentes áreas relacionadas con infraestructura no solamente vial sino para salud, educación, cárceles, entre otras. Según Carlos Cruz, ex ministro de Obras Públicas, "hasta principios del 2000, no hubo en el mundo una política de inversión privada tan exitosa como la chilena, un modelo paradigmático e innovativo que superó con creces las metas de la inversión privada de infraestructura pública que el propio gobierno se había propuesto" (citado en Blazevic, 2008: 23).

Como vemos en este caso, la necesidad de coordinar esfuerzos en pos de resolver el problema de infraestructura vial, generó una APP. Desde la mirada estatal, el modelo de concesiones como APP tiene su justificación dentro de una perspectiva eficientista donde el Estado, al reconocer la situación de déficit, busca nuevas formas de financiamiento para la inversión pública. Los privados por otro lado, 
ven en esta oportunidad un ámbito de negocio nuevo para ellos. Esta situación actúa como detonante de innovación en la gestión pública, principalmente en lo que respecta a la posibilidad de adecuar las prácticas y procedimientos históricos del ministerio respecto de su responsabilidad sobre el diseño, la construcción y la mantención de las obras viales, por un cambio hacia un rol de monitoreo y fiscalización, que claramente puede ser concebido como novedoso, en el sentido planteado por los autores presentados en las secciones previas. La APP actúa como un generador de cambios en las formas en que se llevaba a cabo una tarea por parte del Estado, particularmente el Ministerio de Obras Públicas. Un aspecto interesante de este caso, fue la aparición de casos de corrupción, tales como el MOP-GATE donde se generaron innovaciones que superaron con creces los marcos jurídicos pertinentes y aplicables a la situación, debido al cambio sustantivo que se estaba generando, particularmente en materias de remuneraciones. En palabras de Waissbluth (2005:77):

"En el fondo, fue esta sobrecarga y la imposibilidad de tener directivos adecuadamente remunerados para administrar esta complejidad lo que llevó a la crisis organizacional. Por dar una idea, el salario de un directivo del MOP, que administra contratos por cientos de millones de dólares, era y sigue siendo unas 3 a 5 veces menor que el salario de sus contrapartes en empresas constructoras. Eso llevó al diseño de mecanismos de pago de sobresueldos consistentes básicamente en la ampliación de contratos a firmas proveedoras, que después derivaban esos recursos excedentarios a algunas empresas u organizaciones (como GATE) que administraban el pago de dichos sobresueldos, con el agravante de que eran ingresos personales que no pagaban impuestos. Según el derecho administrativo chileno el uso de partidas presupuestarias con fines distintos a los previstos constituye fraude al fisco, por más loables que fueran los destinos de esos recursos. En el mejor de los casos, estos procedimientos fueron conducidos en el desorden más absoluto, y en el peor, pueden haber generado espacios para la comisión de delitos". 
Como se puede apreciar en este caso, y el análisis propio del mismo, queda de manifiesto que el desarrollo de APP en las concesiones fue un generador de innovaciones. Lamentablemente, al parecer, de acuerdo a la arista judicial del caso, se tradujo en acciones distantes de los preceptos normativos que regulan el sector público chileno.

\section{El Programa Inglés Abre Puertas ${ }^{1}$ : Las APP como generadoras de INNOVACIÓN EN EDUCACIÓN Y FORMACIÓN DE CAPITAL HUMANO}

El Programa Inglés Abre Puertas (PIAP) es creado en el año 2004 por el Ministerio de Educación, con el objetivo de optimizar tanto la enseńanza como el aprendizaje del Inglés en estudiantes de establecimientos educaciones subvencionados a lo largo del país. En términos generales, el objetivo de este programa es capacitar a los estudiantes, principalmente los más desfavorecidos, para que una vez egresados de la enseñanza secundaria cuenten con el inglés como instrumento clave en el desarrollo profesional. El desarrollo de este programa ha sufrido cambios y modificaciones de acuerdo a los liderazgos y contextos políticos en los cuales este programa se ha desarrollado. De este modo, se puede clasificar el programa en tres grandes etapas.

\section{i) Etapa de diseńo (2004-2006)}

Donde se concibe la idea y objetivo principal del programa, ideado desde sus inicios basado en una estructura de tipo horizontal para actuar con mayores niveles de autonomía, propiciando un terreno fértil para una concepción organizacional basada en alianzas público-privadas. De hecho, por esta razón es que la dirección del programa se lleva desde el gabinete del Ministro de Educación de la época, Sergio Bitar, quien también es un actor clave en la formulación del Programa. El director de este período, Horacio Walker, académico, investigador y experto en educación, sienta las bases conceptuales y alcances del programa desde un punto de vista más relacionado con el diseño académico.

1 La información inicial generada para el análisis del Programa Inglés Abre Puertas (PIAP) forma parte de un proyecto de investigación financiado por la Vicerrectoría de Investigación de la Universidad de Chile, a través del programa U-Apoya. Los autores del presente artículo forman parte del equipo de investigación de este proyecto, liderado por la Prof. Verónica Figueroa. 


\section{ii) Etapa de Articulación (2006-2010)}

Es en este período donde se toma conciencia de la necesidad de más recursos y menos trabas burocráticas para alcanzar los objetivos. Por esta razón se escoge la figura de las APP como estrategia central en la gestión del PIAP. El rol del director, Rodrigo Fábrega, ingeniero comercial y profesional del mundo privado con enfoque en los asuntos públicos, es fundamental para entender la articulación y configuración que el programa tiene hasta el momento. Para involucrar a empresas privadas y ONG se generaron instrumentos de validación y verificación de los resultados del programa con el fin de convencer a las organizaciones participantes. Es en esta etapa donde se generan las siguientes estrategias que articulan el programa, donde la participación de organizaciones no estatales es el centro de las iniciativas: i) Programa de Voluntarios Angloparlantes, ii) Programa de Docentes, y, iii) Programa de Practicantes.

En primer lugar, el Programa de Voluntarios tiene como objetivo “(...) administrar y gestionar el área de voluntarios para mejorar la enseñanza del inglés, para dar mayor oportunidad a estudiantes para que aprendan inglés, promover intercambios con otras culturas y trabajar colaborativamente con los profesores de inglés en la enseñanza práctica del inglés" (PIAP, 2008:6). Este programa es administrado por el Centro Nacional de Voluntarios del Ministerio de Educación y el PNUD, y con representación del Ministerio de Educación, Empresas e Instituciones de educación. El objetivo principal de este Centro es propiciar la participación del sector público y privado en la promoción de la enseńanza del inglés e instalar voluntarios, sean docentes, practicantes y/o profesionales angloparlantes que desean colaborar con el objetivo de este programa, hacer de Chile un país bilingüe. El Programa trabaja con metodologías de la enseñanza del inglés creadas en conjunto con la American University, la Universidad Diego Portales y la Universidad Católica además del apoyo de las embajadas de países de habla inglesa para la búsqueda de voluntarios en estos países, el Ministerio de Relaciones Exteriores, la Agencia de Cooperación Internacional, y del sector privado, PRO Chile, SOFOFA y la CPC.

En segundo lugar, el Programa de Docentes tiene como objetivos promocionar la venida a Chile de docentes angloparlantes que deseen en forma voluntaria o pagada ofrecer sus servicios profesionales en establecimientos educacionales dispuestos 
a pagar sus gastos. El Centro Nacional de Voluntarios está a cargo de la gestión y administración de la venida a Chile de estos profesionales.

En tercer lugar, el Programa de Practicantes, a través del Centro, está a cargo de la promoción y coordinación a Chile de profesionales dispuestos a realizar sus prácticas profesionales en una empresa o institución chilena. Estos profesionales son egresados de universidades de preferencia angloparlantes que buscan tener una experiencia profesional en Chile. Básicamente este programa ha sido diseñado para incorporar a las instituciones privadas con y sin fines de lucro a profesionales con un alto dominio del idioma inglés.

\section{iii) Fase de evaluación (2010 - hasta la fecha)}

Desde el cambio de gobierno en 2010, el programa ha experimentado cambios sustanciales en la organización interna y en el lugar que este ocupa en la política pública de educación. El seguimiento del PIAP fue transferido desde el gabinete ministerial a la Unidad de Evaluación y Currículum del Ministerio de Educación. El objetivo de esta nueva etapa está centrado en la calidad, para lo cual se está construyendo diferentes dispositivos de evaluación de los resultados del programa.

$\mathrm{Al}$ analizar el PIAP es posible concluir que este tipo de APP está enmarcado en un modelo más cercano al enfoque de gobernanza, presentado inicialmente en el presente trabajo. En este contexto, y a partir de los análisis iniciales del caso, podemos entender esta APP como el tipo de innovación que genera impacto tanto en las organizaciones como en los contextos extraorganizacionales. El mejor modelo para explicar esta APP como gatillante de innovación es el que nos entrega Barlett y Dibben (2002), el cual hemos explicado anteriormente. Este modelo se enfoca en la persona como agente gatillador de la innovación, donde existe un actor (o actores) con una mentalidad de emprendedor, con ciertas cualidades personales. Como vimos anteriormente la figura del Innovation Champion, como actor al interior de la organización que gatilla la innovación, está presente en la figura de diferentes actores relevantes en el desarrollo del programa, particularmente el Director del Programa del segundo período, Rodrigo Fábrega, quien impulsa, en el marco del desarrollo del proyecto, ya desarrollado como APP, la innovación para mejorar el servicio público que se estaba tratando de entregar. Por otro lado, la figura del Innovation Sponsor, en la persona del Ministro Sergio Bitar, es 
aquella persona que entrega el mandato y soporte político para llevar a cabo esta innovación. Claramente, las presiones intra o extraorganizacionales no eran tan gravitantes para gatillar la innovación en la figura de este programa, como el peso de estos dos personajes, quienes lo propician.

Una de las evidencias de la innovación que gatilla esta APP en el sector público se presenta en la gestión financiera. Por ejemplo, específicamente en la región de Antofagasta, la Fundación Minera La Escondida participa en la aplicación de este programa en conjunto con establecimientos educacionales municipalizados y particulares subvencionados de Antofagasta, con el fin de mejorar los niveles del aprendizaje del inglés y de generar alternativas de desarrollo profesional para los docentes de inglés de la región, financiando una cuarta parte de esta iniciativa (Fundación Minera la Escondida, 2010). Lo anterior ha producido la generación de nuevos instrumentos de gestión y evaluación de los recursos, que no son solamente públicos sino privados, por lo cual la gestión de estos tienen una modalidad especial. Junto con esto, la Fundación Minera La Escondida participa en la implementación de ciertas actividades de este programa como proyectos concursables, pasantías o becas de pedagogía en inglés, entre otros, lo cual ha presionado al sector público a innovar en la generación de mecanismos de seguimiento de gestión que puedan ajustarse a la intervención privada en la resolución de problemas públicos.

Junto con lo anterior, otro factor que podría explicar la APP como gatillante de innovación es la necesidad de la obtención de recursos de una forma más ágil, como se explicó anteriormente. En este sentido, y siguiendo a Osborne y Brown (2005), la innovación surge para contrarrestar la burocracia y generar dispositivos institucionales que permitan una gestión más expedita de los recursos para alcanzar los resultados esperados. Del mismo modo, la interacción con nuevos actores, a partir de la noción de "lazos débiles" ya desarrollada previamente, podría sumar a redes ya establecidas, tales como las propias de los ministerios sectoriales, nuevos actores que entregan nuevas ideas para la solución de viejos problemas. Del mismo modo, esto se transforma en una presión desde el medio para generar cambios en los ministerios, como el de Educación por ejemplo, para adoptar nuevos procesos y diseñar nuevas rutinas más acordes a las dinámicas generadas en la APP creada. 


\section{Conclusiones}

Los nuevos arreglos institucionales entre el sector privado y el gobierno son cada día una realidad más concreta. En el presente documento se pretendió relacionar estos nuevos arreglos institucionales con un tema que ha adornado de manera vasta la literatura de las Ciencias de la administración en general, y de la gestión pública en particular. A través de la revisión de la literatura se pudo caracterizar las APP y la innovación en la gestión pública, focalizando nuestra atención en la posibilidad de la APP como gatilladora de innovación.

Los dos casos presentados en el presente documento, al menos de manera exploratoria, buscan especificar esta relación y dar cuenta de los tipos de innovación que se han presentado. En términos generales podemos ver cómo las APP han logrado generar innovación en los servicios públicos a través del desarrollo de nuevos servicios, cambios innovadores en la gestión de los servicios públicos, y finalmente como espacios de interacción para el nacimiento y desarrollo de nuevos programas, a partir del intercambio de ideas por la confluencia de actores con prismas y visiones diferentes, aglutinados en torno a una misma intención.

Respecto de la entrega de nuevos servicios a la comunidad, parece que el ideario planteado por Moore (1995) respecto de la creación de valor público, sirve como marco teórico para entender la explosión por innovar en nuevos servicios. Las APP en este sentido, ayudan a que esas nuevas ideas o esos nuevos proyectos puedan desarrollarse, consolidando la noción de que el sector público debe o puede dedicarse a proveer más que a producir servicios (Hood, 1991). Las APP pueden ayudar a presionar por nuevos servicios, o por mejorar la calidad y cantidad de los ya entregados, a partir de la suma de esfuerzos en pos del objetivo común que justifica la alianza. Particularmente pertinente es el caso del PIAP para ejemplificar este punto, en tanto nuevas acciones se acometieron a partir de la alianza.

Otro factor que puede inferirse de los casos anteriormente estudiados que generaría un ambiente propicio a la innovación mediante las APP, está relacionado con la búsqueda por parte de los gerentes públicos de soluciones más expeditas, y la superación de trabas burocráticas para la obtención de los resultados esperados. En este sentido, las APP permiten que ciertos procesos se generen bajo el marco jurídico del sector privado, el cual tiene menos trabas que el gubernamental. No 
obstante, en este punto existe una amenaza al accountability público sobre estas formas de arreglos institucionales (Bexell y Mörth, 2010), ya que los contratos entre las diferentes partes son muy complejos, sobre todo en los casos de infraestructura pública, y no son ampliamente publicitados, generando zonas oscuras en la gestión de recursos públicos, que como vimos en el caso de las concesiones del MOP, ha presentado más de un problema.

Un tercer factor que propiciaría un campo fértil para este tipo de alianzas es la visión y el liderazgo de los gerentes públicos así como de políticos en áreas determinadas de política pública. Esta capacidad amplia de visión permitiría a los directivos y/o políticos sumar actores tanto del mundo público como privado, interesados en la solución de problemas públicos a través de la generación de redes, lo cual, como vimos, podría generar innovación en la gestión pública. En este sentido las redes generan una nueva modalidad de intervención intersectorial, lo que es una innovación en sí misma. El establecimiento de mesas intersectoriales, mesas tripartitas, entre otros, son dispositivos que promueven la creación de intrumentos nuevos de gestión pública que permiten coordinar las acciones, sean estas relativas a la gestión, a las finanzas, al control, entre otras. Sin embargo, una de las principales dificultades de esta forma de surgir innovación es la continuidad de las APP. Si no se establecen mecanismos orientados a institucionalizar estos arreglos en el largo plazo, cuando suceden cambios de autoridades políticas, o los llamados ciclos políticos que mencionaba Borins (2001), que no tengan esta visión o liderazgo, o sencillamente que no compartan el proyecto que implica la APP, los impactos de estas innovaciones se podrían ver reducidos.

Las interrogantes que abre este estudio para ser respondidas por investigaciones posteriores tienen relación con descubrir los elementos que permiten sostener esta capacidad de gatillar innovaciones en el tiempo, evitando que los que forman parte de la APP vuelvan a ciclos de estancamiento más que innovación. En este sentido, ¿pueden aprender las APP o pueden desarrollar capacidades de administración del conocimiento? Otra interrogante que es importante resolver a través de futuras investigaciones dice relación con el verdadero impacto en la o las organizaciones involucradas en APP por el hecho de formar parte de la alianza. En otras palabras, sería interesante analizar si las innovaciones internas generadas a partir de la APP son adoptadas por quienes forman parte de la organización pública y no las ven como externas o ajenas a su realidad. Finalmente, sería relevante 
para este ámbito disciplinario, desarrollar investigaciones en torno a las diferencias entre sectores respecto de la posibilidad de gatillar innovaciones. ¿Tiene el mismo efecto generador de innovaciones una APP en el sector social o en el económico? Esto resulta interesante, ya que al parecer la materia sobre la cual la APP actúa debería tener algún grado de influencia en las dinámicas institucionales desarrolladas. En definitiva, las investigaciones deberían orientarse a comprender, manejar y proyectar las innovaciones en la gestión pública, en contextos de APP.

\section{REFERENCIAS BibliográficAS}

Baldock, J. y A. Evers (1991). "On social innovation -an introduction”, en R. Kraan et al. (eds), Care for the Ederly. Significant Innovations in Three European Countries. Frankfurt: Campus Verla.

Barlett, D. y P. Dibben (2002). "Public sector innovation and entrepreneurship: Case studies from local government”. Local Government Studies Vol. 28, No 4, pp. 107-21.

Bexell, M y Mörth, U. (Eds.) (2010). Democracy and Public-Private Partnerships in Global Governance. UK: Palgrave Macmillan.

Bitran, E., y Villena, M. (2010). "El nuevo desafío de las concesiones de obras públicas en Chile”. Revista Estudios Públicos, No 117, pp. 175-217.

Blazevic, H. (2008). Análisis critico de la politica de concesiones viales en Chile: Balances y aprendizajes. Santiago: Estudio de Caso No 102. Magíster Gestión y Políticas Públicas.

Borins, S. (2001). "Public management innovation: toward a global perspective". American Review of Public Administration Vol. 31, No 1, pp. 5-21.

Castells, M. (1992). The Informational City: Economic Restructuring and Urban Development. UK: Wiley-Blackwell.

Considine, M.; Lewis, J. y Alexander, D. (2009). Networks, innovation and public policiy. UK: Palgrave Macmillan.

Damanpour, F. (1991). "Organizational innovation: A meta-analysis of effects of determinants and moderators”. Academy of Management Journal Vol. 34, No. 3, pp. 555-590.

Devlin, R. y G. Moguillansky (2009). Alianzas público-privadas para una nueva visión estratégica del desarrollo. Santiago: CEPAL.

Donahue, J. D., y Zeckhauser, R. J. (2011). Collaborative Governance: Private Roles for Public Goals in Turbulent Times. Princeton UniversityPress. 
Fundación Minera La Escondida (2010). Informe de Sustentabilidad 2010. www.fme.cl/descargas/...reporte-de-sustentabilidad.../download.html (visitada el 29 de marzo de 2012).

Granovetter, M. (1973). “The Strenght of Weak Ties”. American Journal of Sociology, Vol. 78, No 6, pp. $1360-1380$.

Heinrich, C. Lynn Jr, L. y Milward, H. (2009). "A state of agents? Sharpening the debate and evidence over the extent and impact of the trasnformation of governance". Journal of Public Administration Research and Theory Vol. 20 (suppl 1), pp. i3-i19.

Hodge, G. y C. Greve (2005). The Challenge of Public-Private Partnerships: Learning From International Experience Edward Elgar Publishing.

Hood,C. (1991). “A Public Management for all season?” Public Administration Vol. 69, pp. 3-19.

Kooiman, J. (1993). Modern Governance. London, England: SAGE.

Kooiman, J. (2003). Governing as Governance. UK: SAGE.

Koppenjan, J., y Klijn, E. (2004). Managing uncertainties in networks.UK: Routledge.

Korhonen, J. (2003). "On the Ethics of Corporate Social Responsibility: Considering the Paradigm of Industrial Metabolism”. Journal of Business Ethics Vol. 48, No 4, pp. 301-315.

Milward, B. y K. Provan (2000). "Governing the Hollow State". Journal Public Administration Research and Theory Vol. 10, No2, pp. 359-380.

Moore, M. (1997). Creating Public Value: Strategic Management in Government. Harvard University Press.

Newman, J.; Raine, J. y Skelcher, C. (2001). "Transforming local government: innovation and modernization". Public Money and Management, Vol. 21 No 2, pp. 261-68.

Osborne, S, y Brown, K. (2005). Managing change and Innovation in Public Service Organizations. USA: Routledge.

Programa Inglés Abre Puertas (2008). Informe Centro Nacional de Voluntarios. Chile: Ministerio de Educación. Obtenido de: www.educacionempresa.cl/archivos/CNV\%20comision\%207. doc

Rittel, H. y Webber, M. (1973). “Dilemmas in a General Theory of Planning”. Policiy Sciences, Vol. 4, pp. 155-169.

Roberts, N. y P. King (1996). Transforming Public Policy. Dynamics of Public Entrepreneurships and innovation. San Francisco: Jossey-Bass,

Robinson, H.; Carrillo, P; Anumba, C. J. y Patel, M. (2010). Governance and Knowledge Management for Public-Private Partnerships. UK: Wiley-Blackwell. 
Savas, E. (1990). "A Taxonomy of Privatization Strategies". Policy Studies Review, Vol. 18, No 2, pp. 110-128.

Sorensen, E. y Torfing, J. (2011). Enhancing Collaboration Innovation in Public Sector. Administration and Society Vol. 43, No 2, pp. 842-868.

Torres, G. (2004). ¿Burócratas e Innovadores? Revista del CLAD Reforma y Democracia No 29, pp. $1-12$.

Waissbluth, M. (2005). “La Reforma del Estado en Chile 1990-2005”. Serie Gestión No 76. Departamento Ingeniería Industrial. Universidad de Chile.

Walker, R.M. (2006). "Innovation type and diffusion: An empirical analysis of local government". Public Administration, Vol. 84, No 2, pp. 311-335.

Wettenhall, R. (2003). “The Rhetoric and Reality of Public-Private Partnerships”. Public Organization Review Vol. 3, No 1, pp. 77-107.

Williamson, O. (1988). “The Logic of Economic Organization”. Journal of Law, Economics, \& Organization Vol. 4, No 1, pp. 65-93. 